\title{
Variables Related to Iron Status and Genetic Background Among Korean Populations: Ironing Out the Differences
}

\author{
Paulo Caleb Junior Lima Santos ${ }^{1}$
}

Published online: 15 July 2015

(c) Springer Science+Business Media New York 2015

Laboratory evidence of elevated iron stores can be an important predictor of cardiovascular diseases, diabetes, neurodegenerative diseases, and cancers [1-3]. Iron status is routinely evaluated by biochemical assays such as serum iron (SI), total iron-binding capacity (TIBC), and serum ferritin (SF). Although SF has high sensitivity for iron overload, it has low specificity, since inflammatory processes, liver diseases, and alcohol consumption can elevate SF. Transferrin saturation (TS), which is calculated by dividing SI by TIBC, identifies iron overload as well, with $\mathrm{TS} \geq 50 \%$ for females or $\geq 60 \%$ for males suggestive of genetic disease [4].

TS is elevated in many studies of Korean populations [5-7] although the most commonly reported genetic predisposition to iron overload, the hereditary hemochromatosis (HH)-associated p.Cys282Tyr homozygous or compound heterozygous (p.Cys282Tyr/p.His63Asp) genotypes, is rare in Korean populations. In this issue of Digestive Diseases and Sciences, Oh et al. [7] thus sought, using an epidemiological approach based on the Fifth Korea National Health and Nutrition Examination Survey (KNHANES V-1 2010), to determine whether an alternative genetic basis for the observed elevated TS exists. They reported whether TS patterns cluster within families, concluding that a greater percentage of Koreans had increased TS compared to European populations [7].

Paulo Caleb Junior Lima Santos

pacaleb@usp.br

1 Laboratory of Genetics and Molecular Cardiology, Heart Institute (InCor), University of São Paulo Medical School, Av. Dr. Enéas de Carvalho Aguiar, 44 Cerqueira César, São Paulo CEP 05403-000, Brazil
Oh et al. included 260 families with 370 children in their analysis. Parents and children were classified into groups based on TS values. The mean TS value of the offspring differed according to the parental groups, whereas age, daily iron intake, and aspartate and alanine transaminases did not affect TS [7], even though aging, daily iron intake, and liver tests are reportedly influential [8-10]. The absence of association of these factors with TS might have been due to the inclusion criteria used, since subjects were excluded if they were anemic, if they had serum transaminase concentrations $>40 \mathrm{U} / \mathrm{L}$, or if the specimens used for SI measurements were not obtained at a standardized time. Although these criteria minimized the variability of their data, the authors might have chosen some of these inclusion criteria to evaluate the possibility that those variables were not the cause of the high TS in Koreans. Furthermore, to evaluate the relationship between iron status and the studied variables, additional iron status tests such as the SF and soluble transferrin receptor should have been measured to further define iron status.

The authors concluded that the findings suggest unidentified genetic variations underlie high TS in Koreans beyond the gene encoding the human hemochromatosis protein (HFE) p.Cys282Tyr and p.His63Asp polymorphisms. The p.His63Asp variant, frequent in many countries (allelic frequency of approximately $15 \%$ ), has been associated with iron status measurements in individuals from general population [11-13]. These significant associations were not associated with iron overload, but with slightly higher mean values. Although the authors did not genotype the population for $H F E$, these $H F E$ variants as stated previously are rare in Korean populations. Thus, one can assume that $H F E$ variants did not affect the iron status of Koreans, at least no more so than in populations of European ancestry. The authors concluded that iron status 
is based on a polygenic profile, which contributes $20-30 \%$ of the variation of blood iron measures [14].

Genome-wide association, familial, and exome sequencing studies reported significant correlations between polymorphisms and iron status or the severity of the HH phenotype $[15,16]$. For example, Benyamin et al. [15] analyzed the genetic data and biochemical iron status published in 11 European population-based studies in 48,972 subjects, identifying genome-wide significant loci including several well-known iron-related genes (i.e., $H F E$, SLC4OA1, TF, TFR2, TFRC, TMPRSS6) and other novel genes (i.e., ABO, ARNTL, FADS2, NAT2, TEX14). In another study, the PCSK7 rs236918 variant was reported as a risk factor for the development of liver cirrhosis in patients with $\mathrm{HH}$ carrying the homozygous genotype for HFE p.Cys282Tyr [16].

In conclusion, the authors present a compelling case that iron load observed in Korean populations is likely genetically based due to several gene loci besides HFE. Which genes are involved in which combinations, and the clinical consequences of their polymorphism or mutation await further studies.

\section{References}

1. Fonseca-Nunes A, Jakszyn P, Agudo A. Iron and cancer risk-a systematic review and meta-analysis of the epidemiological evidence. Cancer Epidemiol Biomarkers Prev. 2014;23:12-31.

2. Orban E, Schwab S, Thorand B, et al. Association of iron indices and type 2 diabetes: a meta-analysis of observational studies. Diabetes Metab Res Rev. 2014;30:372-394.

3. Nandar W, Connor JR. HFE gene variants affect iron in the brain. J Nutr. 2011;141:729S-739S.
4. Brissot P, Troadec MB, Bardou-Jacquet E, et al. Current approach to hemochromatosis. Blood Rev. 2008;22:195-210.

5. Choi SJ, Min WK, Chun S, et al. Frequencies of C282Y and H63D mutations and transferrin saturation indices in the Korean population. Clin Chem Lab Med. 2002;40:689-692.

6. Lee SH, Kim JW, Shin SH, et al. HFE gene mutations, serum ferritin level, transferrin saturation, and their clinical correlates in a Korean population. Dig Dis Sci. 2009;54:879-886.

7. Oh SH, Jeong TD, Lee W, et al. Analysis of familial tendencies in transferrin saturation in a Korean population. Dig Dis Sci. (Epub ahead of print). doi:10.1007/s10620-015-3720-y.

8. Casgrain A, Collings R, Harvey LJ, et al. Effect of iron intake on iron status: a systematic review and meta-analysis of randomized controlled trials. Am J Clin Nutr. 2012;96:768-780.

9. Olsson KS, Ritter B, Lundin PM. Liver affection in iron overload studied with serum ferritin and serum aminotransferases. Acta Med Scand. 1985;217:79-84.

10. $\mathrm{Xu} \mathrm{J}$, Knutson MD, Carter CS, et al. Iron accumulation with age, oxidative stress and functional decline. PLoS One. 2008;3:e2865.

11. Santos PC, Krieger JE, Pereira AC. Molecular diagnostic and pathogenesis of hereditary hemochromatosis. Int J Mol Sci. 2012;13:1497-1511.

12. Santos PC, Cancado RD, Terada CT, et al. HFE gene mutations and iron status of Brazilian blood donors. Braz J Med Biol Res. 2010;43:107-114.

13. Jackson HA, Carter $\mathrm{K}$, Darke $\mathrm{C}$, et al. HFE mutations, iron deficiency and overload in 10,500 blood donors. Br J Haematol. 2001;114:474-484.

14. Njajou OT, Alizadeh BZ, Aulchenko Y, et al. Heritability of serum iron, ferritin and transferrin saturation in a genetically isolated population, the Erasmus Rucphen Family (ERF) Study. Hum Hered. 2006;61:222-228.

15. Benyamin B, Esko T, Ried JS, et al. Novel loci affecting iron homeostasis and their effects in individuals at risk for hemochromatosis. Nat Commun. 2014;5:4926.

16. Stickel F, Buch S, Zoller H, et al. Evaluation of genome-wide loci of iron metabolism in hereditary hemochromatosis identifies PCSK7 as a host risk factor of liver cirrhosis. Hum Mol Genet. 2014;23:3883-3890. 